\title{
3D culture of Erdheim-Chester disease tissues unveils histiocyte metabolism as a new therapeutic target
}

Erdheim-Chester disease (ECD) is a rare non-Langerhans cell histiocytosis, characterised by tissue infiltration by foamy CD $68^{+}$ CD1a ${ }^{-}$histiocytes. ${ }^{1}$ The disease has pleomorphic clinical manifestations, including long bones and extraskeletal involvement, and may be life-threatening, particularly when heart and central nervous system are affected. ${ }^{1}$

ECD histiocytes secrete proinflammatory cytokines ${ }^{2}$ and carry activating mutations along the RAS-RAF-MEK-ERK protein kinase signalling pathway, most commonly the $\mathrm{BRAF}^{\mathrm{V} 600 \mathrm{E}}$ oncogenic mutation. ${ }^{34}$ Accordingly, patients with ECD have been treated with cytokine inhibitors, including infliximab, ${ }^{15}$ and, more recently, with the BRAF ${ }^{\mathrm{V} 600 \mathrm{E}}$ inhibitor vemurafenib. ${ }^{6}$ The latter, however, induces sustained but partial clinical responses and recurrences on discontinuation, ${ }^{6}$ underlining the need for more effective therapeutic strategies.

To identify the outcomes downstream constitutive ERK phosphorylation in ECD histiocytes and their response to small molecule-based inhibition, we performed three-dimensional (3D) culture of tissues from three $\mathrm{BRAF}^{\mathrm{V} 600 \mathrm{E}}$-mutated $\mathrm{ECD}$ patients in the RCCS bioreactor ${ }^{7}$ (and online supplementary methods) in the presence/absence of vemurafenib or infliximab, used as control.

All patient samples maintained production of prototypical cytokines and chemokines ${ }^{2}$ in bioreactor, thus validating this technology also for ECD; moreover, infliximab, and, to a lesser degree, vemurafenib, significantly decreased cyto-chemokines and soluble tumour necrosis factor (TNF) receptor (sTNF-R) levels (figure 1A), remarkably mirroring the downmodulation reported in treated patients ${ }^{5}$ (and data not shown).

Immunohistochemistry (IHC) on ECD tissues in bioreactor showed preserved viability, histoarchitecture and expression of histiocyte lineage and activation markers (figure 1B) for up to 6 days. Vemurafenib specifically targeted mutated ECD histiocytes, as demonstrated by decreased ERK phosphorylation, without affecting viability or persistence of $\mathrm{CD} 68^{+}$cells (figure $1 \mathrm{~B}$ and online supplementary figure S1). Conversely, the drug decreased TNF- $\alpha$ production (figure $1 \mathrm{~B}, \mathrm{f}, \mathrm{f}^{1}$ ) paralleling the significantly reduced cytokine release in supernatants (figure 1A).

Cancer metabolism has gained renewed and growing interest, being reprogrammed glucose metabolism from oxidative phosphorylation to aerobic glycolysis and increased lactate production the best characterised metabolic phenotype. ${ }^{8}$ Oncogenic BRAF 
A

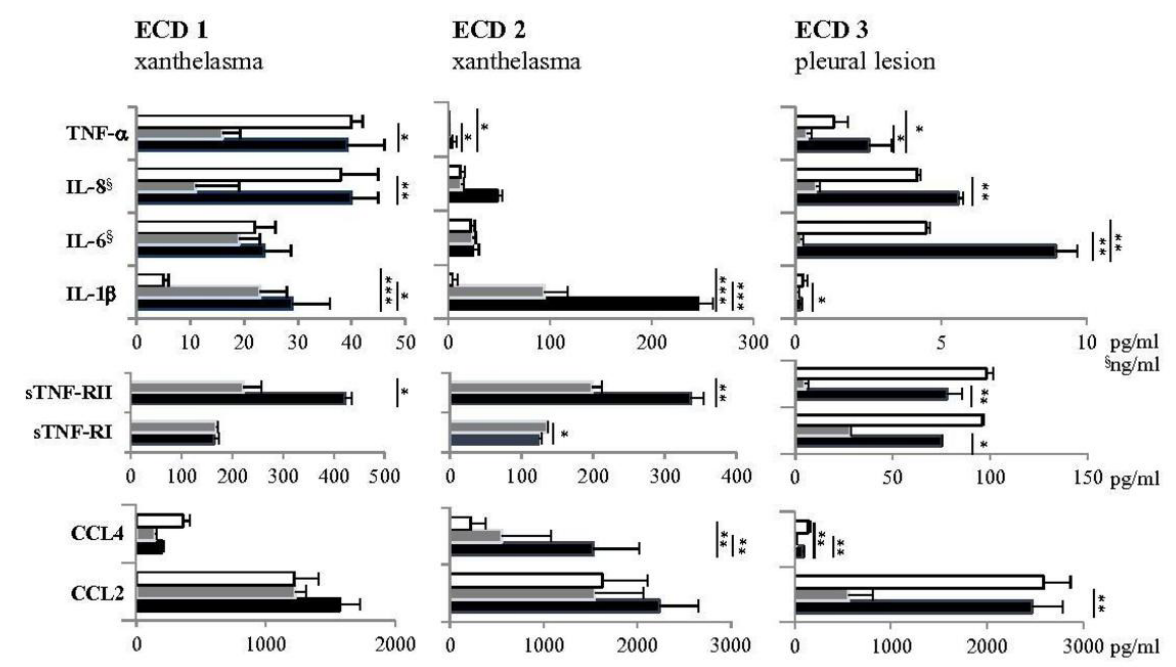

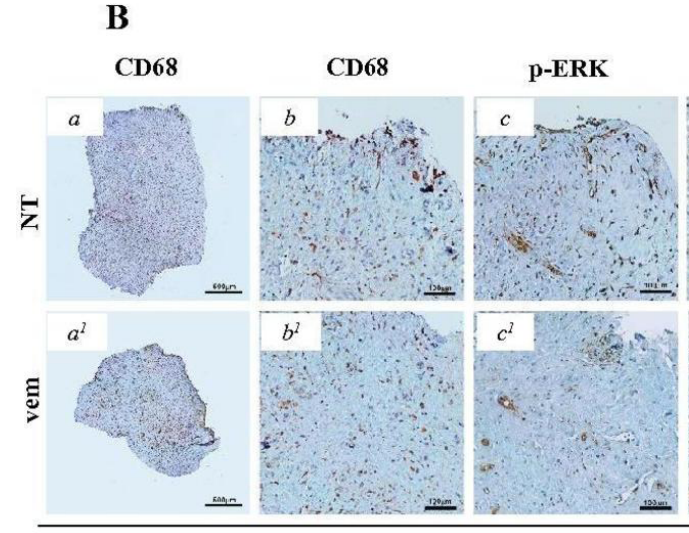

day 2
Glut-1

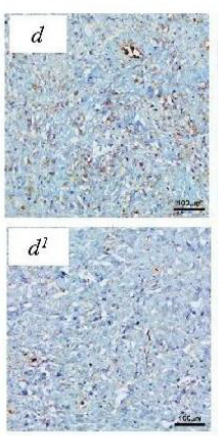

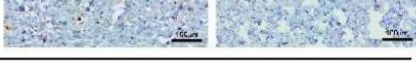

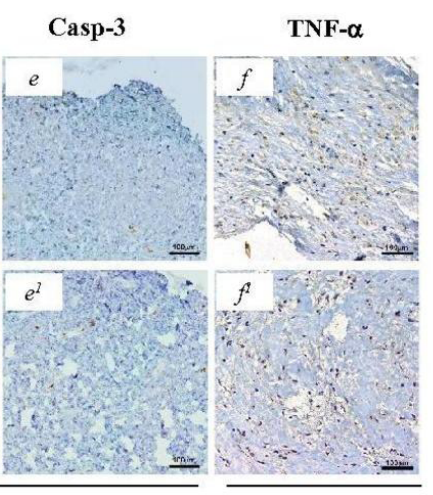

day 6
C
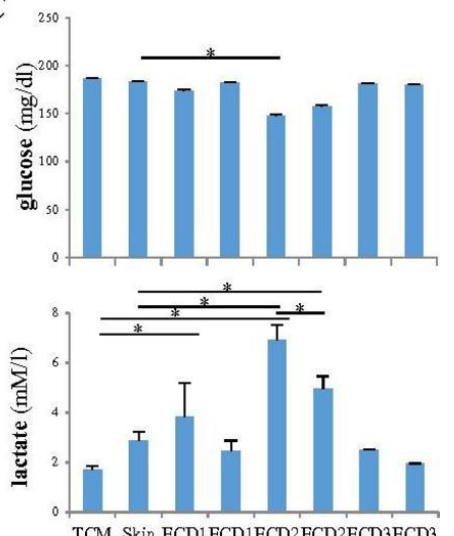

TCM Skin ECD1ECD1ECD2ECD2ECD3ECD3
NT Vem NT Vem NT Vem

Figure 1 Vemurafenib treatment affects glycolytic metabolism in Erdheim-Chester disease (ECD) tissues cultured in bioreactor. (A) Tissues from three patients with ECD were cultured in bioreactor in the absence (black bars) or presence of either the tumour necrosis factor (TNF) inhibitor infliximab (10 $\mu \mathrm{g} / \mathrm{mL}$, grey bars) or the BRAF ${ }^{\mathrm{V} 600 \mathrm{E}}$ inhibitor vemurafenib $(6 \mu \mathrm{M}$, white bars). Concentrations of cytokines, chemokines and soluble TNF receptors (sTNF-R) I and II (the latter bona fide expression of TNF activity ${ }^{5}$ ) were determined in day 2 culture supernatants by Bio-Plex Multiplex Cytokine assay and ELISA assay, respectively. Data are means \pm SD of triplicate values. (B) ECD pleural fragments from patient 3 were cultured in the presence/absence of vemurafenib (vem, $6 \mu \mathrm{M})$. Samples were then retrieved at day $2\left(a-e, a^{1}-e^{1}\right)$ and day $6\left(f^{1}, f^{1}\right)$, fixed and submitted to immunohistochemistry (IHC). Bars represent 500 and $100 \mu \mathrm{m}$. (C) Glucose (upper) and lactate (lower) concentrations were determined in the supernatants from ECD tissues in bioreactor in the presence/absence of vemurafenib (vem, $6 \mu \mathrm{M}$ ) and from normal skin samples as a control. Data are means $\pm S D$ of triplicate values. Statistical analysis was performed using Student's $t$-test. ${ }^{*} \mathrm{P} \leq 0.05$; ${ }^{*} \mathrm{P} \leq 0.01$; ${ }^{* *} \mathrm{P} \leq 0.001$. Casp-3, caspase-3; NT, untreated; p-ERK, phospho-ERK; TCM, tissue culture medium.

affects glucose metabolism by multiple mechanisms, including upregulation of the glucose transporter Glut- $1 .{ }^{8}$ Glut-1 is also upregulated by hypoxia and inflammation in rheumatic and cardiovascular diseases. ${ }^{9}$ Accordingly, a fraction of $\mathrm{CD} 68^{+}$ECD histiocytes expressed Glut-1, as shown by IHC (online supplementary figure S2A,B) and fluorescence-activated cell sorting analysis (online supplementary figure $\mathrm{S} 2 \mathrm{C}$ ), and retained its expression in culture in bioreactor (figure 1B,d). Given the unique opportunity to assess tissue metabolism through metabolite determination in bioreactor supernatants, we determined lactate levels, which were increased, compared with basal levels and normal skin samples (figure 1C). Notably, vemurafenib downmodulated both Glut-1 expression (figure $1 \mathrm{~B}, \mathrm{~d}^{1}$ ) and lactate production (figure $1 \mathrm{C}$ ).

Altogether, we here demonstrate, as a proof of concept, that the bioreactor technology allows investigating ECD pathophysiology and response to drugs, thus overcoming the lack of cell lines and suitable animal models. Significantly, upregulated Glut-1 expression and lactate production, and its reversal on vemurafenib treatment, delineate aerobic glycolysis as a novel BRAF-driven feature of ECD histiocytes. Since Glut-1 is the transporter for the radiotracer of ${ }^{18} \mathrm{~F}$-fluorodeoxyglucose (FDG-PET), these data may provide the molecular basis for the well-known efficacy of the technique in monitoring response to therapy in $\mathrm{ECD} .{ }^{1}$

Although not proliferating, ${ }^{2}$ ECD histiocytes may experience increased energy requirement for the production of immune mediators, as for inflammatory macrophages. In bioreactor, vemurafenib decreased their cytokine/chemokine release, conceivably because of impaired metabolism, but not their viability, at variance with other BRAF-mutated tumours. The outcome of vemurafenib treatment in ECD histiocytes may depend on the activation of adaptive responses to energy deprivation, including autophagy, or, conversely, on detrimental features (hypoxia, acidosis, limited nutrients availability) of the native microenvironment, possibly overcome by our culture conditions in bioreactor. ${ }^{7}$ 
Further exploitation of the 3D culture system is needed to identify and harness metabolic signalling and supporting adaptive responses in mutated histiocytes, in the perspective of designing new therapeutic strategies for patients with ECD.

\section{Antonello Villa, ${ }^{1}$ Daniela Belloni, ${ }^{2}$ Barbara Vergani, ${ }^{1}$ Simone Cenci, ${ }^{3}$ Giulio Cavalli, ${ }^{4,5}$ Riccardo Biavasco, ${ }^{5,6}$ Monica Rodolfo, ${ }^{7}$ Maria Giulia Cangi, ${ }^{8}$ Claudio Doglioni, ${ }^{5,8}$ Lorenzo Dagna, ${ }^{4,5}$ Elisabetta Ferrero, ${ }^{2}$ Marina Ferrarini ${ }^{2}$}

${ }^{1}$ Consorzio MIA, University of Milano-Bicocca, Milan, Italy

${ }^{2}$ Division of Experimental Oncology, San Raffaele Scientific Institute, Milan, Italy

${ }^{3}$ Division of Genetics and Cell Biology, San Raffaele Scientific Institute, Milan, Italy

${ }^{4}$ Unit of Immunology, Rheumatology, Allergy and Rare Diseases, San Raffaele

Scientific Institute, Milan, Italy

${ }^{5}$ Vita-Salute University, Milan, Italy

${ }^{6}$ San Raffaele Telethon Institute for Gene Therapy (SR-Tiget), San Raffaele Scientific Institute, Milan, Italy

${ }^{7}$ Department of Experimental Oncology and Molecular Medicine, IRCCS, Istituto Nazionale per Cura dei Tumori, Milan, Italy

${ }^{8}$ Pathology Unit, San Raffaele Scientific Institute, Milan, Italy

Correspondence to Marina Ferrarini, Division of Experimental Oncology, San Raffaele Scientific Institute, Milan 20132, Italy; ferrarini.marina@hsr.it

\section{Handling editor Josef Smolen}

Acknowledgements The authors thank Licia Rivoltini and Giovanna Musco for critical support and advices, and Mauro Motta, Marta Strollo and Silvia Heltai for technical assistance. The authors also thank Kathy Brewer and the ECD Global Alliance (DeRidder, LA) for their unceasing support.

Contributors $A V$, EF and MF designed the study. DB and BV performed the experiments and collected the data. CD reviewed the histological samples. MR provided experimental tools. MGC determined the BRAF status. DB conducted the statistical analysis. AV, SC, GC, RB, EF and MF analysed and interpreted the data. GC collected clinical information. LD diagnosed and followed patients with ECD and contributed to discussion. AV, EF and MF wrote the manuscript. All authors critically reviewed and approved the final version of the manuscript.

Funding This study was funded by the ECD Global Alliance and by the Italian Ministry of Health (grant number GR-2009-1594586).

Competing interests None declared.
Patient consent Obtained.

(C) Author(s) (or their employer(s)) 2019. No commercial re-use. See rights and permissions. Published by BMJ.

LD, EF and MF contributed equally.

$$
\text { Check for updates }
$$

To cite Villa A, Belloni D, Vergani B, et al. Ann Rheum Dis 2019;78:862-864.

Received 12 September 2018

Revised 14 November 2018

Accepted 20 November 2018

Published Online First 28 November 2018

Ann Rheum Dis 2019;78:862-864. doi:10.1136/annrheumdis-2018-214432

\section{REFERENCES}

1 Diamond EL, Dagna L, Hyman DM, et al. Consensus guidelines for the diagnosis and clinical management of Erdheim-Chester disease. Blood 2014;124:483-92.

2 Stoppacciaro A, Ferrarini M, Salmaggi C, et al. Immunohistochemical evidence of a cytokine and chemokine network in three patients with Erdheim-Chester disease: implications for pathogenesis. Arthritis Rheum 2006;54:4018-22.

3 Haroche J, Charlotte F, Arnaud L, et al. High prevalence of BRAF V600E mutations in Erdheim-Chester disease but not in other non-Langerhans cell histiocytoses. Blood 2012;120:2700-3.

4 Cangi MG, Biavasco R, Cavalli G, et al. BRAFV600E-mutation is invariably present and associated to oncogene-induced senescence in Erdheim-Chester disease. Ann Rheum Dis 2015;74:1596-602.

5 Dagna L, Corti A, Langheim S, et al. Tumor necrosis factor $\alpha$ as a master regulator of inflammation in Erdheim-Chester disease: rationale for the treatment of patients with infliximab. J Clin Oncol 2012;30:e286-e290.

6 Haroche J, Cohen-Aubart F, Emile JF, et al. Reproducible and sustained efficacy of targeted therapy with vemurafenib in patients with BRAF(V600E)-mutated ErdheimChester disease. J Clin Oncol 2015;33:411-8.

7 Ferrarini M, Steimberg N, Ponzoni M, et al. Ex-vivo dynamic 3-D culture of human tissues in the RCCSTM bioreactor allows the study of Multiple Myeloma biology and response to therapy. PLoS One 2013;8:e71613.

8 Hay N. Reprogramming glucose metabolism in cancer: can it be exploited for cancer therapy? Nat Rev Cancer 2016;16:635-49.

9 Biniecka M, Canavan M, McGarry T, et al. Dysregulated bioenergetics: a key regulator of joint inflammation. Ann Rheum Dis 2016;75:2192-200. 